\section{PSICOLOGIA IBEROAMERICANA}

\section{Psicología lberoamericana}

ISSN: 1405-0943

psicología.iberoamericana@uia.mx

Universidad Iberoamericana, Ciudad de

México

México

Espinosa Rodríguez, Emmanuel; Mayoral Sánchez, Edwin G.; Laca Arocena, Francisco A. Altruismo y bienestar social en la explicación de voluntariado en estudiantes mexicanos de

bachillerato y licenciatura

Psicología Iberoamericana, vol. 21, núm. 2, julio-diciembre, 2013, pp. 85-95

Universidad Iberoamericana, Ciudad de México

Distrito Federal, México

Disponible en: http://www.redalyc.org/articulo.oa?id=133930525009

Cómo citar el artículo

- Número completo

- Más información del artículo

- Página de la revista en redalyc.org

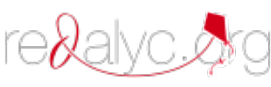

Sistema de Información Científica

Red de Revistas Científicas de América Latina, el Caribe, España y Portugal

Proyecto académico sin fines de lucro, desarrollado bajo la iniciativa de acceso abierto 


\title{
Altruismo y bienestar social en la explicación de voluntariado en estudiantes mexicanos de bachillerato y licenciatura
}

\author{
Altruism and Social Well-Being in the Explanation of Volunteerism \\ among Mexican High-School and University Students
}

\author{
Emmanuel Espinosa Rodríguez* \\ Edwin G. Mayoral Sánchez** \\ Francisco A. Laca Arocena*** \\ Universidad de Colima, Colima, México
}

\section{RESUMEN}

El objetivo del presente estudio fue examinar la relación entre el bienestar social y el altruismo para explicar el voluntariado en estudiantes mexicanos. Se utilizó una muestra de 1028 estudiantes, 514 de bachillerato y 514 de licenciatura, entre quince y treinta años de edad. Los participantes contestaron una serie de medidas de autoinforme: Escalas de Bienestar Social de Blanco \& Díaz (2005), Escala de Altruismo de Corral, García, Castro, Viramontes \& Limones (2010), y se validó el Cuestionario de Intereses sobre Trabajo Voluntario. Mediante un modelo multivariante se encontró que el altruismo es una variable mediadora entre el bienestar social y el voluntariado. Se discuten los resultados y se señalan las limitaciones e implicaciones de la investigación.

Palabras clave: voluntariado, altruismo, bienestar social, jóvenes mexicanos, psicología positiva.

\section{ABSTRACT}

This study seeks to examine the relationship between social well-being and altruism to explain volunteerism among Mexican students. A sample was taken of 1028 students (514 high school students and 514 undergraduates) aged between 15 and 30. The participants answered a series of self-report measures: Scales of Social Well-Being by Blanco \& Díaz (2005), Scale of Altruism by Corral, García, Castro, Viramontes \& Limones (2010). The Questionnaire of Interest in Volunteer Work was also validated. The multivariant model showed that altruism is a variable mediator between social well-being and volunteerism. We discuss the results and indicate the limitations and implications of the research.

Keywords: volunteerism, altruism, social well-being, young Mexicans, positive psychology.

\footnotetext{
* Recibido: 22 de enero, 2013 - Aceptado: 2 de agosto, 2013

Correspondencia: Emmanuel Espinosa Rodríguez, Programa de Estudiantes Voluntarios (EVUC), Universidad de Colima, Av. Universidad 333, colonia Las Víboras, Colima, C. P. 28040.Tel. 0131231611 40.em_espinosa@ucol.mx

** Correspondencia: Edwin G. Mayoral Sánchez, Centro Universitario de Investigaciones Sociales, Universidad de Colima. Av. Gonzalo de Sandoval, colonia Las Víboras, Colima, C. P. 28040. Tel. 0131231611 27.edwin_mayoral@ucol.mx y e_mayoral182@hotmail.com

*** Correspondencia: Francisco A. Laca Arocena, Facultad de Psicología, Universidad de Colima, Av. Universidad 333, colonia Las Víboras, Colima, C. P. 28040. Tel. 0131231610 91. francisco_laca@ucol.mx
} 


\section{INTRODUCCIÓN}

El voluntariado tiene su origen en antiguas costumbres de solidaridad comunal, por ejemplo, el control y la prevención de los incendios forestales y la protección de la vida silvestre (Alfaro, 1993). Se define voluntariado como un comportamiento prosocial planificado que se desarrolla, generalmente, dentro de una organización, beneficia a otras personas, a animales o al medio ambiente; implica un considerable gasto personal de tiempo, de energía y en ocasiones de dinero (Castro, 2002; Clary et al., 1998; Dávila, 2009). De acuerdo con las características de los participantes y de la actividad voluntaria se podrá valorar el grado de colaboración, el involucramiento, el compromiso y la satisfacción con el voluntariado (Clary et al., 1998; Dávila, 2009). El trabajo voluntario está cambiando de su vertiente tradicional a una moderna, es decir, en la actualidad es más común encontrar voluntarios informales que formales. El tradicional o formal se enmarca en una estructura organizacional consolidada, como puede ser una comunidad política o religiosa (Tienen, Scheepers, Reitsma \& Schilderman, 2011), supone un compromiso a largo plazo y con frecuencia está basado en la membresía, en la que el altruismo constituye un factor fundamental para la cooperación (Rehberg, 2005). El moderno o informal consiste en la realización de proyectos temporales y específicos, en el que está definido el tipo de participación; en este sentido, se ha encontrado que éste se explica por la motivación individual (Tienen et al., 2011).

Además de las variables individuales, la cultura parece ejercer un gran efecto en la conducta voluntaria. Los países europeos y anglosajones tienen las tasas de voluntariado más altas, así como una profunda línea de investigación sobre el tema (Galán \& Cabrera, 2002; Janoski, Musick \& Wilson, 1998; Marta, Pozzi \& Marzana, 2010). El posmaterialismo propuesto por Inglehart (1998) ayudaría a entender el fenómeno en el que una sociedad más desarrollada alcanza un cierto nivel de seguridad personal, que se refleja en un mayor interés por las relaciones interpersonales, la estética y la solidaridad. A raíz de la actual crisis económica existe una expansión y un interés renovado por el voluntariado, ya que éste tiene repercusiones sociales y culturales de largo alcance. Un ejemplo reciente de esto es la Cumbre mundial de innovación en el voluntariado, celebrada en Madrid, España, en marzo de 2012.

Los datos de los voluntarios que asisten a las organizaciones no gubernamentales (ONG) están fragmentados, por lo que en México se carece de información empírica sistemática, y es urgente realizar estudios sobre éstos en cualquiera de sus vertientes psicosociales. A lo largo de su historia, el voluntariado mexicano ha desempeñado un papel más bien asistencialista, siendo predominante el auxilio de las mujeres (sobre todo de clase alta) como un gesto caritativo y dadivoso hacia los demás (Serna, 2010), aunque esta tendencia está cambiando con la diversidad de instituciones y de voluntarios en el país. Por otra parte, en situaciones de conflicto social, como la guerra contra el narcotráfico emprendida en México desde 2006, los niveles de confianza y cohesión social se reducen considerablemente, lo que erosiona la acción voluntaria. Así lo indica una reciente investigación llevada a cabo con jóvenes mexicanos en la que se aprecia una tendencia hacia el individualismo y la indiferencia social (Laca, Mejía, Mayoral \& Yáñez, 2012), lo que puede disminuir la motivación voluntaria en la juventud, etapa del desarrollo que constituye el pilar del voluntariado.

Es común leer que el voluntariado es posible gracias a relaciones de solidaridad y cohesión social; sin embargo, en la revisión de la literatura no se encontró ningún estudio que considerara al bienestar social como una variable central para la explicación de la conducta voluntaria. A esto se añade la poca investigación que distingue qué variables psicológicas están implicadas en el voluntariado en estudiantes mexicanos. Por ello, el presente estudio surge del interés de conocer la posible relación existente entre el bienestar social y el altruismo para explicar el voluntariado en ámbito estudiantil.

Se define bienestar social como el autorreporte de un individuo sobre la calidad de sus relaciones con otras personas, su grupo cercano y su comunidad (Keyes \& Shapiro, 2004). El bienestar social se compone de distintos elementos que, en conjunto, muestran en qué medida un individuo está funcionando de manera óptima en su entorno social (por ejemplo, como vecinos, compañeros de trabajo y ciudadanos). Un sentido de bienestar social surge del conocimiento de que la propia vida es útil y constructiva para el 
bien común (Bauer \& Park, 2010). La investigación de Keyes (1998) constituye una aproximación importante al estudio del bienestar psicológico, y ha proporcionado una estructura social para conceptualizar el funcionamiento positivo. Lo que había faltado en la literatura del bienestar subjetivo, según Keyes (1998), es el reconocimiento de que las personas pueden evaluar la calidad de sus vidas y el funcionamiento personal desafiando los criterios sociales. Dada la independencia de las medidas tradicionales del bienestar subjetivo, el bienestar social es un importante marcador de la calidad de vida de un país.

Por su parte, el altruismo es considerado como una conducta prosocial; se cree que está relacionado con la valoración que hace el individuo de las circunstancias para el mejor funcionamiento de la sociedad. Batson (1998) lo define como la unión de conductas que hacen los seres humanos a favor de los demás, sin obtener beneficio por ello. El altruismo ha sido vinculado en la psicología a la prosociabilidad y al comportamiento de ayuda, en su mayoría a nivel interpersonal (Batson, 1991), pero se ha dirigido menor atención a las esferas intergrupales, comunitarias o sociales (Stürmer, Snyder, Kropp \& Siem, 2006).

\section{MÉTODO}

\section{Participantes}

La muestra estuvo compuesta por 1028 participantes de bachillerato y licenciatura de la ciudad de Colima, México, de los cuales 514 participan en actividades de voluntariado y 514 no son voluntarios. El rango de edad osciló entre quince y treinta años, con media en 18.7 $(\mathrm{dt}=2.76)$. El 50.3\% estuvo conformado por hombres y $49.7 \%$ por mujeres. La muestra total de los estudiantes que participaron en el estudio fue balanceada en relación con el género y la edad.

\section{Instrumentos}

Escalas de Bienestar Social. Es una adaptación al castellano de Blanco y Díaz (2005), del original de Keyes (1998). Consta de 25 ítems en forma de afirmaciones ("Siento que soy una parte importante de mi comunidad”), de los cuales el participante elige posicionarse en una escala Likert de 1 (Totalmente en desacuerdo) hasta 5 (Totalmente de acuerdo). Se compone de cinco subescalas o factores del bienestar social: integración social ( $\alpha=0.69$, ítems 1 a 5$)$; aceptación social ( $\alpha=0.83$, ítems 6 a 11); contribución social ( $\alpha=0.70$, ítems 12 a 16); actualización social ( $\alpha=0.79$, ítems 17 a 21$)$; y coherencia social ( $\alpha=0.68$, ítems 22 a 25). A excepción de los ítems 1, 2, 3, 4, 12, 16, 19, el resto son inversos, con el propósito de controlar el sesgo de repetición en la respuesta ("Mis actividades diarias no aportan nada que valga la pena a la sociedad"). Los resultados se calcularon con la puntuación total del bienestar social. $\mathrm{El}$ alfa de Cronbach para este estudio fue de 0.86.

Escala de Altruismo de Corral, García, Castro, Viramontes y Limones (2010). Consiste de diez ítems en foma de acciones ("Donar sangre cuando escucha en la radio o televisión que alguna persona necesita del mismo tipo de sangre que usted tiene"), en donde la persona puede posesionarse en una de las cuatro opciones, donde 0 es "Nunca" y 3 "Siempre". La escala mide las acciones que proporcionan beneficios a otros sin la expectativa de recibir nada a cambio, con el fin de aumentar el bienestar de quien o quienes reciben la ayuda. El instrumento tiene un alfa de 0.81 .

Cuestionario de Intereses sobre Trabajo Voluntario. Este instrumento fue diseñado en una muestra de estudiantes del Programa de Estudiantes Voluntarios de la Universidad de Colima (Evuc) por Medina y Verdugo (comunicación personal, 17 de abril de 2012). El cuestionario se compone de ocho ítems en forma de afirmaciones, que evalúan las razones y los motivos para realizar voluntariado ("Porque ayudar a los demás me hace sentir bien"), donde el participante contesta una escala de 1 (Totalmente en desacuerdo) hasta 5 (Totalmente de acuerdo). El alfa de Cronbach resultante en este estudio fue de 0.87 . El análisis factorial se detallará en los resultados.

\section{Procedimiento}

Se solicitó a los participantes su apoyo para contestar intencionalmente los instrumentos, en una aplicación en línea en las salas de cómputo de sus planteles - la mayoría fueron de la Universidad de Colima-, previo acuerdo con las direcciones respectivas. Se optó por este procedimiento porque permitía la captura de datos con fidelidad, evitando errores en la plantilla estadística. Los participantes conocieron el objetivo y el propósito de la investigación y se les garantizó la priva- 
cidad y el uso de los datos sólo para fines científicos. El análisis de éstos se realizó en el spss, versión 17 y en el Programa Amos 6. Se utilizaron las siguientes pruebas estadísticas: análisis factorial exploratorio y sus respectivos indicadores de ajuste (método de extracción: análisis de componentes principales; método de rotación: normalización varimax con Kaiser), prueba t de Student, y análisis de trayectorias.

\section{RESULTADOS}

Se realizó un análisis factorial exploratorio usando el método de extracción de componentes principales con rotación varimax de los grupos de estudiantes voluntarios y no voluntarios, para conocer las propiedades psicométricas del Cuestionario de Intereses sobre Trabajo Voluntario. Se llevó a cabo el test de KaiserMeyer-Olkin (кмо) у la prueba de esfericidad de Bartlett para comprobar la adecuación del muestreo. La medida de кмо fue de 0.89 , la cual fue mayor al valor requerido mínimo de 0.5 . La prueba de esfericidad de Bartlett resultó significativa con un valor de $\mathrm{p}<0.000\left(\chi^{2}=3660.19\right)$, mostrando que la matriz de correlación no es una matriz de identidad. Los anteriores análisis indican que el factorial fue aplicable a la muestra. El método de extracción arrojó una adecuada solución monofactorial, el cual explica $54.06 \%$ de la varianza; al factor se le renombró voluntariado. El análisis de fiabilidad nos muestra que el instrumento tiene un alfa de Cronbach de 0.87. La mayoría de los ítems tienen un peso factorial de 0.70 (varía el rango hasta 0.79 ), siendo 0.81 el valor más alto. Sólo un ítem tuvo un peso factorial de 0.51 , pero se conservó al no afectar significativamente a la varianza y a la fiabilidad del cuestionario. En la tabla 1 se resume el análisis factorial.

En la tabla 2 se muestran las puntuaciones medias en los factores de bienestar social, altruismo y voluntariado. Los resultados indican puntuaciones altas e intermedias en estos constructos. Como puede apreciarse, en general las mujeres puntuaron más alto en bienestar social y voluntariado, tanto en el grupo de voluntarios como en el de no voluntarios. Las puntuaciones en la escala de altruismo fueron prácticamente iguales para hombres y mujeres.

Tabla 1. Factorial exploratorio del Cuestionario de Intereses sobre Trabajo Voluntario

\begin{tabular}{l|c}
\hline Ítems (motivos para ofrecerse como voluntario) & $\begin{array}{c}\text { Factor I } \\
\text { (voluntariado) }\end{array}$ \\
\hline Para aprender cosas nuevas y diferentes & 0.74 \\
\hline Siento que es mi deber ciudadano & 0.74 \\
\hline Porque mis amigos realizan trabajo voluntario & 0.51 \\
\hline Es importante para adquirir experiencia profesional & 0.70 \\
\hline Para estar rodeado de personas que les gusta ayudar a los demás & 0.79 \\
\hline Lo veo como una oportunidad de hacer algo por los demás & 0.81 \\
\hline Quisiera ocupar mi tiempo libre en algo productivo & 0.71 \\
\hline Porque ayudar a los demás me hace sentir bien & 0.80 \\
\hline Varianza explicada & $54.06 \%$ \\
\hline Confiabilidad por alfa de Cronbach & 0.87 \\
\hline
\end{tabular}


Tabla 2. Medias de los participantes en bienestar social, altruismo y voluntariado

\begin{tabular}{c|c|c|c|c}
\hline \multirow{2}{*}{ Total } & \multicolumn{2}{|c|}{ Hombres } & \multicolumn{2}{c}{ Mujeres } \\
\hline$N=1028$ & $\mathrm{Nv}$ & $\mathrm{V}$ & $\mathrm{Nv}$ & $\mathrm{V}$ \\
\hline Bienestar social: (A) & $3.53(0.504)$ & $3.63(0.507)$ & $3.69(0.503)$ & $3.77(0.486)$ \\
\hline Altruismo: (B) & $1.64(0.473)$ & $1.77(0.531)$ & $1.77(0.484)$ & $1.78(0.486)$ \\
\hline Voluntariado: (C) & $3.81(0.756)$ & $3.86(0.865)$ & $4.05(0.599)$ & $4.07(0.623)$ \\
\hline
\end{tabular}

Nota 1: rango de valores para: (A) de 1 a 5; (B) de 0 a 3. (C) de 1 a 5.

Nota 2: Nv: no voluntarios; V: voluntarios.

Para indagar las posibles variables de interés que estén mediando, se procedió a realizar algunas correlaciones. Los resultados muestran que el bienestar social correlaciona significativamente con el altruismo $(\mathrm{r}=$ $0.17, \mathrm{p}<0.01)$ y el voluntariado $(\mathrm{r}=0.19, \mathrm{p}<0.01)$. También se observa significancia estadística entre el altruismo y el voluntariado $(\mathrm{r}=0.38, \mathrm{p}<0.01)$ y bienestar social $(\mathrm{r}=0.17, \mathrm{p}<0.01)$. El voluntariado correlacionó significativamente con el altruismo $(\mathrm{r}=0.38$, $\mathrm{p}<0.01)$ y bienestar social $(\mathrm{r}=0.19, \mathrm{p}<0.01)$.

La muestra estuvo compuesta de 1028 participantes, de los cuales 514 eran estudiantes voluntarios y 514 no voluntarios. Para poder realizar el análisis multivariante denominado análisis de trayectorias, se eliminaron los valores extremos en cada una de las variables de análisis: se descartaron los que estuvieron a \pm 2.5 desviaciones estándar de la media. Así pues, para el análisis de trayectorias la muestra quedó compuesta por 987 participantes, divididos en 491 estudiantes voluntarios y 496 no voluntarios.

Para que el análisis factorial confirmatorio fuera significativo, se esperó que la probabilidad asociada a chi-cuadrada fuera mayor de 0.05 . El problema es que para muestras muy grandes chi-cuadrada puede ser no significativa, aunque el modelo tenga buen ajuste (Lévy \& Varela, 2003). La combinación más utilizada hoy en día, respecto al ajuste del modelo (Boomsma, 2000; McDonald \& Ho, 2002) es el siguiente: chi cuadrada $\left(\mathrm{X}^{2}\right)$, índice de la raíz cuadrada media del error de la aproximación (RMSEA), indicador ilustrativo del ajuste global del modelo (ECVI), índice estandarizado de raíz cuadrada media residual (SRMR), índice de bondad de ajuste (GFI) e índice de ajuste comparativo (CFI).

La figura 1 corresponde al análisis del grupo tanto de voluntarios como de no voluntarios $(\mathrm{n}=987)$. En relación con las medidas de ajuste absoluto, el modelo presenta el siguiente valor para el estadístico de chi-cuadrado, con un nivel de significancia bajo: $\mathrm{X}^{2}=61.395, \mathrm{gl}=1 ; 0.05, \mathrm{p}=0.000$. Sin embargo, debe considerarse que este valor es sensible a los tamaños muestrales y al supuesto de normalidad, lo que implica que ante muestras grandes el valor de este estadístico generalmente resulta significativo, situación que no es deseable en el análisis factorial confirmatorio, por lo que no resulta un buen índice de ajuste en este caso. Como complemento, evaluamos con el índice de bondad del ajuste, cuyo valor resultó alto y, por ende, indicativo de un ajuste aceptable ( $\mathrm{GFI}=0.97)$. Respecto al índice de ajuste comparativo, el resultado también es señal de un ajuste adecuado (CFI $=0.85)$. Finalmente, el índice de la raíz cuadrada media del error de la aproximación fue el siguiente: RMSEA $=0.062$, por lo que indica que la correlación residual media está dentro de los parámetros aceptables y corrobora a un nivel razonable de ajuste.

Los índices de ajuste comparativo (índice de ajuste normado e índice de ajuste incremental), que evalúan el ajuste incremental del modelo, dan cuenta de valores moderados, cercanos a uno $(\mathrm{NFI}=0.85$; IFI $=0.85)$, lo que implica que existe un buen ajuste entre el modelo, reflejando en gran medida la información contenida en los datos. Y por último el índice de bondad de ajuste parsimonioso $(\mathrm{PGFI}=0.090)$ nos indica que el modelo 
ha alcanzado un buen ajuste. Los resultados confirman una influencia indirecta de la variable del bienestar social. De igual modo, la variable altruismo muestra efectos directos con la variable voluntariado.

La tabla 3 contiene las estimaciones de los parámetros del modelo, el error estándar aproximado (SE) y la proporción crítica (CR).

El altruismo es la variable que mejor explica las conductas del voluntariado al presentar una relación fuerte y positiva $(\beta=0.35)$. En la figura 1 se especifica el modelo estructural, el cual cuenta con $21 \%$ de la varianza explicada, por lo que se puede observar que las varianzas de todos los constructos evaluados contribuyen al voluntariado.

Posteriormente, se verificó el mismo análisis para estudiantes voluntarios, en el que se aprecia que el nivel de ajuste absoluto es bueno (RMSEA $=0.056$ ), con un índice de ajuste no normalizado alto (NFI $=0.958)$ $\mathrm{y}$ un índice de ajuste comparativo alto ( $\mathrm{CFI}=0.906 ; \mathrm{X}^{2}$ $=19.995, \mathrm{gl}=1$; con un nivel de significancia bajo 0.05 $(\mathrm{p}=0.000))$. Lo anterior implica que al considerar sólo a los estudiantes voluntarios en el análisis, el modelo se mantiene con un nivel moderado en los índices de ajuste (tabla 4 ).

Tabla 3. Parámetros estimados para el modelo de voluntarios y no voluntarios

\begin{tabular}{|l|c|c|c|c|}
\cline { 3 - 5 } \multicolumn{2}{c|}{} & Parámetro estimado & SE & \multicolumn{2}{c|}{ CR } \\
\hline \multirow{2}{*}{ Altruismo } & Bienestar Social & 0.164 & 0.030 & 5.233 \\
\hline \multirow{2}{*}{ Voluntariado } & Bienestar Social & 0.037 & 0.035 & 1.234 \\
\cline { 2 - 5 } & Altruismo & 0.351 & 0.035 & 12.250 \\
\hline
\end{tabular}

Figura 1. Análisis de trayectorias en estudiantes voluntarios y no voluntarios

Chi cuadrada $=61.395$

Grados de libertad $=1$

Probabilidad $=.000$
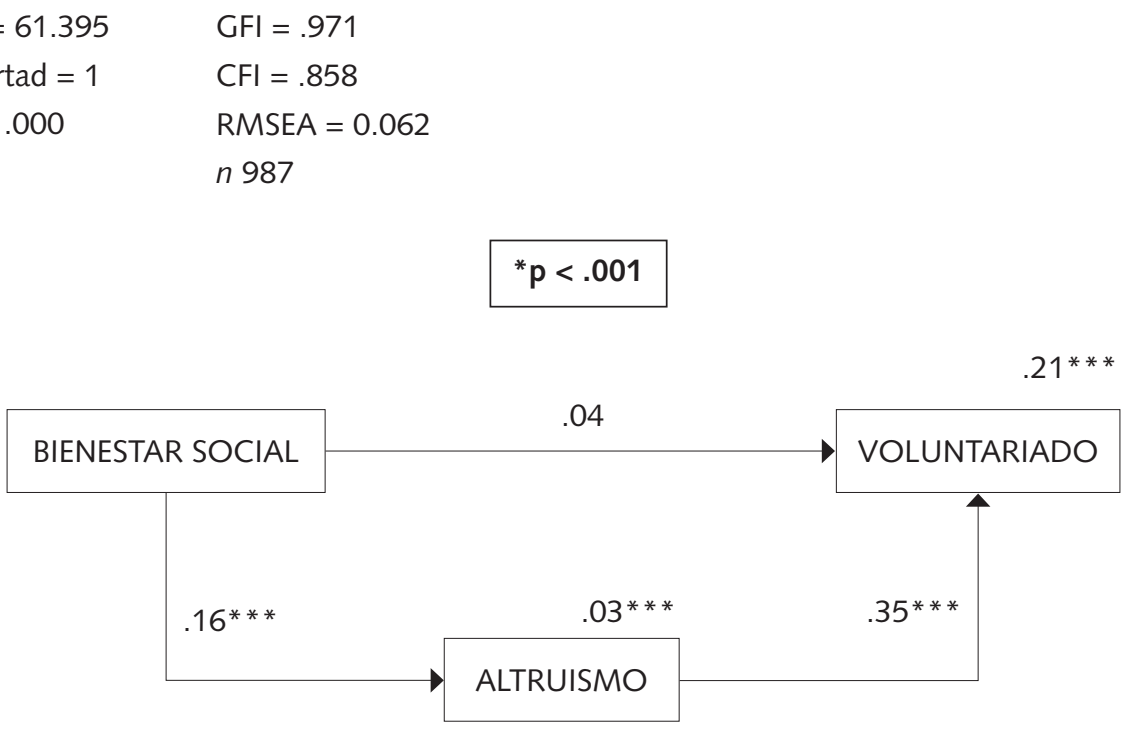
Tabla 4. Ajustes globales del modelo de voluntarios

\begin{tabular}{l|c|c|c}
\hline Medida de ajuste & Modelo & Saturado & Independencia \\
\hline GFI & 0.980 & 1.000 & 0.644 \\
\hline RMSEA & 0.056 & & 0.242 \\
\hline AGFI & 0.955 & & 0.542 \\
\hline RFI & 0.915 & & 0.000 \\
\hline IFI & 0.991 & 1.000 & 0.000 \\
\hline CFI & 0.906 & 1.000 & 0.000 \\
\hline NFI & 0.958 & 1.000 & 0.000 \\
\hline PGFI & 0.138 & & 0.501 \\
\hline
\end{tabular}

Un segundo análisis contempló los resultados sólo con los estudiantes voluntarios (figura 2). El modelo estructural cuenta con $22 \%$ de la varianza explicada, por lo que se puede observar que las varianzas de todos los constructos evaluados contribuyen a las conductas voluntarias. Como puede notarse, ambos modelos tienen adecuados índices de ajuste. Los resultados de los análisis de trayectorias indican que la relación entre el bienestar social y el voluntariado no es lineal, sino que el altruismo media y potencia esta correlación.

\section{DISCUSIÓN}

Se ha cumplido con el objetivo de conocer la relación existente entre las variables de bienestar social y el altruismo para explicar el voluntariado en el ámbito estudiantil. El interés por estudiar las conductas prosociales desde la psicología empírica es relativamente reciente. En este sentido, esta investigación se suma a los pocos trabajos cuantitativos que existen en México acerca de las variables psicológicas implicadas en la explicación del voluntariado.

Figura 2. Análisis de trayectorias en estudiantes voluntarios

Chi cuadrada $=19.995$

Grados de libertad $=1$

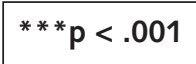

Probabilidad $=.000$

$\mathrm{GFI}=.980$

$\mathrm{CFI}=.906$

RMSEA $=0.056$

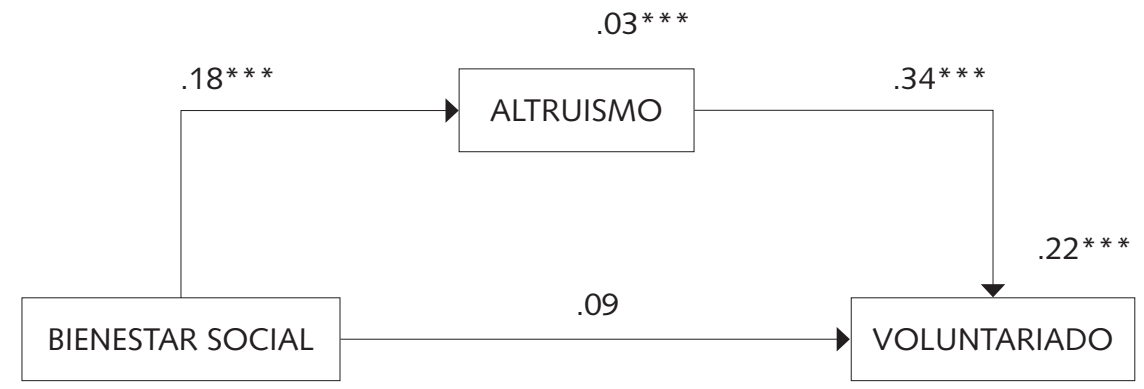


En los resultados, inicialmente interesó realizar un análisis factorial exploratorio del Cuestionario de Intereses sobre Trabajo Voluntario para identificar sus propiedades psicométricas. Los ocho ítems que lo componen convergieron en un factor, al cual se le denominó voluntariado. Dicho instrumento puede ser empleado en este campo, aunque se sugiere seguir utilizándolo en investigaciones futuras para tener mayor certeza de su validez y confiabilidad.

Como puede notarse, la muestra en este estudio fue representativa de los estudiantes de nivel bachillerato y licenciatura de la ciudad de Colima. Fue homogénea en cuanto a género y edad, lo cual facilitó la precisión de los datos obtenidos. Prueba de esto es que en todas las variables las medias resultaron ser altas a intermedias. Sin excepción, las mujeres puntuaron más alto que los hombres en todas las escalas, tanto en voluntarios como en no voluntarios, por lo que el género en sí mismo explica una fuerte tendencia en la participación de actividades voluntarias. Es lugar común en la psicología encontrar en las mujeres prevalencia de las conductas llamadas asistenciales y prosociales, debido en buena parte a la socialización diferenciada a la que se exponen niños y niñas, y a las motivaciones propias del varón y la mujer (Baumeister, 2010); de modo que los hallazgos de este estudio apuntan en la misma dirección. Estos resultados son concordantes con investigaciones que relatan una mayor participación en actividades prosociales de las mujeres y adolescentes (Flanagan, Bowes, Jonsson, Csapo \& Sheblanova, 1998).

Si bien los resultados de los análisis de trayectorias muestran que la relación entre el bienestar social y el voluntariado no es unidireccional, los análisis estructurales señalan que el altruismo media esta relación y es la variable de mayor peso en la explicación del voluntariado. Éste es un indicador habitual de la participación, compromiso cívico y comportamiento prosocial (Keyes, 1998; Keyes \& Shapiro, 2004). En el estudio de Keyes (1998), los niveles de bienestar social fueron más altos entre adultos que han trabajado con otros en sus barrios para resolver algún problema, comparado con adultos que han estado involucrados en sus barrios hace más de un año y aquellos que nunca han participado en dichas actividades. Todas las medidas de bienestar social aumentaron los niveles de seguridad percibida y la integridad de los vecinos se acrecentó.
Sin embargo, para establecer la relación existente de manera positiva entre el bienestar social y el altruismo para aquellos estudiantes que deciden realizar un voluntariado, se debe tomar en cuenta lo siguiente. Las personas están inmersas en estructuras sociales y en comunidades que las llevan a experimentar desafíos e incontables tareas, donde los retos pudieran ser el criterio que utilizan los individuos para evaluar la calidad de vida (Keyes, 1998). En este caso, el bienestar social implica, de alguna manera, un sentido de pertenencia; en general, alude a la medida en que la gente siente que tiene algo en común con los otros que constituyen su entorno social. En otras palabras, se puede referir al constructo de apoyo social, en el que los voluntarios perciben elevados niveles de unificación social; como consecuencia se genera un alto grado de satisfacción con ellos mismos.

En definitiva, es importante destacar que los resultados obtenidos muestran que aunque el bienestar social es importante para explicar el voluntariado, parecen definir una nueva hipótesis en la que el bienestar social, por sí solo, no basta para encauzar los comportamientos del voluntariado. A pesar de que no se involucró la variable de individualismo en este estudio, el creciente individualismo y, particularmente, la percepción y el desánimo que algunos jóvenes experimentan a raíz de la lucha contra el crimen organizado en México (Laca et al., 2012), facilita que los sentimientos de integración vayan debilitándose y persista la sensación de que los demás son unos extraños, ajenos a nuestros valores o nuestro estilo de vida. Entonces, se podría pensar que si el estudiante acepta a la sociedad con sus múltiples aspectos, unos positivos y otros negativos, más razones y motivos tendrá para ofrecerse como voluntario. Para que realice una acción voluntaria debe tener una percepción de que existe un clima emocional donde se sienta aceptado por los demás que confían en él, y que los otros son capaces de procurar amabilidad y cooperación, lo que elevaría el altruismo y favorecería el voluntariado.

Por otro lado, un estudiante que perciba una incongruencia entre lo que recibe económicamente de acuerdo con su nivel educativo, afectará de manera significativa a su autoconcepto, disminuyendo sus niveles de bienestar social percibidos y su disposición al voluntariado. 
Las raíces para entender el altruismo son más complejas de lo que se pensaba anteriormente (Warneken \& Tomasello, 2009), por lo que se volvió un tema de estudio multidisciplinario. En laboratorios de psicología estadounidenses y alemanes, como es el caso del de Felix Warneken (Harvard University, Department of Psychology) y el de Michael Tomasello (Max Planck Institute for Evolutionary Anthropology, Department of Developmental and Comparative Psychology), algunas de las líneas de investigación más recientes o en boga en este campo versan sobre el entendimiento de las conductas prosociales en niños y chimpancés. Por ello, existen investigaciones que analizan cuáles son los mejores motivadores de las conductas altruistas y en qué momento se podrían desarrollar, aunado al intrigante problema de cuándo una conducta es altruista y cuándo no lo es (Warneken, 2009). Las definiciones conceptuales más recientes de altruismo involucran términos que anteriormente se consideraban independientes: ayudar a otros a lograr sus metas (la persona que ayuda actúa como si ella fuera la responsable directa), compartir cosas valiosas, como por ejemplo comida y víveres, e informar sobre cosas que necesitan saber. "En una analogía económica, podría de manera altruista proveer a otros con bienes, servicios o información" (Warneken \& Tomasello, 2009, p. 397).

Los retos de la investigación futura en este campo en México, y en general en Iberoamérica, son profundos; requieren la evaluación de constructos cercanos y análisis más puntuales que ayuden a explorar las variables que están implicadas en el voluntariado. Por ejemplo, la empatía podría ser una variable importante a testar en futuros estudios. A partir del descubrimiento de las neuronas espejo (las responsables de la empatía), se viene concibiendo desde perspectivas multidisciplinarias, que la empatía es un ingrediente esencial para la cooperación, presente no sólo en humanos, sino en otras especies como los primates, o en general en animales con un cerebro evolucionado (De Waal, 2004; López, Valdovinos, Méndez-Díaz \& Mendoza-Fernández, 2009). Teniendo las neuronas espejo un fundamento biológico, no está de más encontrar factores comunes psicológicos y sociales en la explicación de la empatía, con la esperanza de ensanchar la comprensión de las conductas prosociales. Las personas empáticas tienen la capacidad de reconocer las emociones ajenas para reaccionar a señales sociales sutiles, indicativas de qué necesitan o quieren los otros (Davis, 1996). La empatía hace a las personas más aptas para el desempeño de las conductas prosociales y, en consecuencia, facilitaría la conducta voluntaria. La reacción empática se torna en una preocupación por el bienestar de otro individuo, ya que se mezclan sentimientos de simpatía, compasión y rabia al presenciar el sufrimiento y la injusticia ajena (Batson, 1991; Mestre, Samper \& Frías, 2002).

En gran parte de los estratos sociales y regiones de México es necesaria la conducta solidaria y voluntaria para, literalmente, salvarle la vida a alguien. México es un país oprimido cada vez más por la pobreza - la estadística más baja estima que cincuenta millones de mexicanos viven en esta situación, de los cuales veinte millones son clasificados como pobres en extremo; los datos fehacientes son oscuros en este sentido- $-y$ dada su ubicación geográfica, también es susceptible de desastres naturales. Es importante conocer qué variables están implicadas en el voluntariado y en el altruismo, ya que son factores protectores en caso de urgencias o necesidades para algún grupo social o población en riesgo. Las instituciones que formalmente se encargan de ayudar a los demás son insuficientes para la población que compone el país. La creencia en las instituciones políticas y clericales es más débil que nunca, por lo que hay que reforzar a la sociedad civil mediante el apoyo mutuo y concertado. 


\section{REFERENCIAS}

Alfaro, J. (1993). Elementos para una definición de la psicología comunitaria. En Olave, L. \& Zambrano, L. (Eds.), Psicología comunitaria y salud mental en Chile (pp. 14-31). Santiago de Chile: Universidad Diego Portales.

Batson, C. D. (1998). Altruism and prosocial behavior. En Gilbert, D. T., Fiske, S. T. \& Lindzey, G. (Eds.), The handbook of social psychology (4ta. Ed.), 2 (pp. 282316). New York: McGraw Hill.

Batson, C. D. (1991). The altruism question: toward a social-psychological answer. Hillsdale: Erlbaum.

Bauer, J. J. \& Park, S. W. (2010). Growth is not just for the young: growth narratives, eudaimonic resilience, and the aging self. En Frye, P. S. \& Keyes, C. L. M. (Eds.), New frontiers in resilient aging: life-strengths and wellbeing in late life (pp. 60-89). Cambridge: Cambridge University Press.

Baumeister, R. F. (2010). Is there anything good about men?: How cultures flourish by exploiting men. New York: Oxford University Press.

Blanco, A. \& Díaz, D. (2005). El bienestar social: su concepto y medición. Psicothema, 17(4), 582-589.

Boomsma, A. (2000). Reporting analyses of covariance structures. Structural Equation Modeling, 7(3), 461-483.

Castro, R. de (2002). Voluntariado, altruismo y participación activa en la conservación del medio ambiente. Intervención Psicosocial, 11(3), 317-331.

Clary, E. G., Snyder, M., Ridge, R. D., Copeland, J., Stukas, A. A., Haugen, J. \& Miene, P. (1998). Understanding and assessing the motivations of volunteers: a functional approach. Journal of Personality and Social Psychology, 74(6), 1516-1530.

Corral, V., García, C. H., Castro, L., Viramontes, I. \& Limones, R. (2010). Equity and sustainable lifestyles. En Corral, V., García, C. H. \& Frías, M. (Eds.), Psychological approaches to sustainability: current trends in theory, research and applications (pp. 185-204). New York: Nova Science Publishers.

Dávila, M. C. (2009). Assessment of the volunteer process model in enviromental volunteers. Interamerican Journal of Psychology, 43(1), 181-186.

Davis, M. H. (1996). Empathy: a social psychological approach. Boulder: Westview Press.

De Waal, F. B. M. (2004). On the possibility of animal empathy. En Manstead, A. S. R., Frijda, N. \& Fischer,
A. (Eds.), Feelings and emotions: The Amsterdam Symposium (pp. 381-401). New York: Cambridge University Press.

Flanagan, C. A., Bowes, J. M., Jonsson, B., Csapo, B. \& Sheblanova, E. (1998). Ties that bind: correlates of adolescents' civic commitments in seven countries. Journal of Social Issues, 54(3), 457-475.

Galán, E. \& Cabrera, P. (2002). Características personales y madurez del voluntariado. Revista de Psicodidáctica, $14,59-70$.

Inglehart, R. (1998). Modernización y postmodernización. el cambio cultural, económico y político en 43 sociedades. Madrid: Centro de Investigaciones Sociológicas.

Janoski, T., Musick, M. \& Wilson, J. (1998). Being volunteered? The impact of social participation and prosocial attitudes on volunteering. Sociological Forum, 13(3), 495-519.

Keyes, C. L. M. (1998). Social well-being. Social Psychology Quarterly, 61(2), 121-140.

Keyes, C. L. M. \& Shapiro, A. D. (2004). Social well-being in the United States: a descriptive epidemiology. En Brim, O. G., Ryff, C. D. \& Kessler, R. C. (Eds.), How healthy are we? A national study of well-being at midlife (pp. 350-372). Chicago: University of Chicago Press.

Laca, F. A., Mejía, J. C., Mayoral, E. G. \& Yáñez, C. L. (2012). Violence related attitudes, horizontal individualism and collectivism in young mexicans. Psychology Journal, 9(1), 2-12.

Lévy, J. P. \& Varela, J. (Comps). (2003). Análisis multivariable para las ciencias sociales. Madrid: Prentice Hall.

López, D. I., Valdovinos, A., Méndez-Díaz, M. \& Mendoza-Fernández, V. (2009). El sistema límbico y las emociones: empatía en humanos y primates. Psicología Iberoamericana, 17(2), 60-69.

Marta, E., Pozzi, M. \& Marzana, D. (2010). Volunteers and ex-volunteers: paths to civic engagement through volunteerism. Psykhe, 19(2), 5-17.

McDonald, R. P. \& Ho, M. H. R. (2002). Principles and practice in reporting structural equation analyses. Psychological Methods, 7(1), 64-82.

Mestre, M. V., Samper, P. \& Frías, M. D. (2002). Procesos cognitivos y emocionales predictores de la conducta prosocial y agresiva: la empatía como factor modulador. Psicothema, 14(2), 227-232. 
Rehberg, W. (2005). Altruistic individualists: motivations for international volunteering among young adults in Switzerland. Voluntas: International Journal of Voluntary and Nonprofit Organizations, 16(2), 109-122.

Serna, M. G. (2010). La diversidad y el contexto cambiante del voluntariado en México. Espiral, 16(47), 141-172.

Stürmer, S., Snyder, M., Kropp, A. \& Siem, B. (2006). Empathy-motivated helping: the moderating role of group membership. Personality and Social Psychology Bulletin, 32(7), 943-956.
Tienen, M. v., Scheepers, P., Reitsma, J. \& Schilderman, H. (2011). The role of religiosity for formal and informal volunteering in the Netherlands. Voluntas: International Journal of Voluntary and Nonprofit Organizations, 22(3), 365-389.

Warneken, F. (2009). Digging deeper: a response to commentaries on "The roots of human altruism". British Journal of Psychology, 100, 487-490.

Warneken, F. \& Tomasello, M. (2009). Varieties of altruism in children and chimpanzees. Trends in Cognitive Sciences, 13(9), 397-400. 